\title{
Analyzing the Structure of the EGOV Conference Community
}

\author{
Nuša Erman and Ljupčo Todorovski \\ Univeristy of Ljubljana, Faculty of Administration \\ Gosarjeva 5, SI-1000 Ljubljana, Slovenia \\ \{nusa.erman, ljupco.todorovski\}@fu.uni-1j.si
}

\begin{abstract}
The paper applies social network analysis techniques to the task of analysis the dynamics and structure of the e-government research community. From the bibliographic data about papers published in the proceedings of this conference (International Conference on e-Government), we build a coauthorship network representing collaboration patterns among community members in the period from 2005 to 2009 . The co-authorship network analysis helps us identify the most productive and central authors in EGR community, as well as delineate the community structures through finding its sub-groups and core parts. In this way, several sub-communities are revealed in sense of the thematic topics, affiliations, and geographical origins of authors.
\end{abstract}

Keywords: e-government research analysis, social network analysis, coauthorship network, scientific community, e-government research community.

\section{Introduction}

e-Government research (EGR) as a scientific field has already been a subject of study from different points of view. Authors have considered the maturity $[8,9]$ and the development [10] of the field, have studied the use of different methodological approaches [1], have compared various definitions and limitations of the egovernment concept [17], or have profiled the EGR community [6]. A serious common limitation of these studies is that they take a wide variety of different approaches, which often prohibits comparison and/or unification of the obtained results into a single map of the EGR field. In our previous study [7], we proposed the use of social network analysis (SNA) as a general methodology commonly used in scientometrics and bibliometrics and applied to many different scientific fields (see, e.g., [13,3]). Paper presents the results of applying SNA to the study the citation network, induced from the papers published at this (EGOV) conference. The results of the study identify the most influential authors and relate them to the thematic topics that prevailed the EGOV conference through the four years from 2005 to 2008.

In this paper, we continue the work presented in [7] with changing the focus of the analysis from citation networks to networks of collaboration within the EGR community. Let us first define the concept of scientific community. As sociology of science argues, the most significant aspect of every scientific community is the idea of communication between scientists, which represents the foundation of the scientific 
community. In particular, we can define scientific community as the totality of working, interacting, and knowledge sharing scientists that share common and standardized procedures of scientific communication. The scientific community is often quantified through the analysis of scientific publications, and consecutively through the citation, co-citation, and co-authorship analysis [11]. At this point, the methods of scientometrics and bibliometrics are usually applied. Based on the scientific publications, these methods measure scientific activities through different levels of aggregation, enabling the analysis of research collaborations, evolution of scientific fields, and corresponding scientific networks [15].

In a related study of the EGR scientific community [14], author analyzes the data gathered in the e-Government Master Library of references ${ }^{1}$. Using self-defined criteria, author defines core journals, core conferences, and core researchers in the community. Furthermore, taking into account number of publications, author identifies most prolific researchers, their disciplines, research methods used, and the outlets of core parts of EGR community. In another absorbing attempt of EGR community analysis [6], author identifies the most productive authors, their gender, area of academic expertise, and background, as well as a calculus of papers with different number of co-authors, leading research universities, geographical regions, paper types, paradigm, and research methods which are present in the papers published in the journal "Transforming Government: People, Process and Policy".

In this paper, we introduce alternative approach to the study of scientific communities, which on top of identifying most active individuals, takes into consideration structural properties of whole EGR community. To do so, we apply SNA methodologies to the network of co-authorship in the papers, published in the proceedings of the EGOV conference in the period of eight years from 2002 to 2009. The nodes in the co-authorship networks represent authors and the edges represent joint articles published in the proceedings of the EGOV conference. The analysis of this network helps us identify the most productive but also the most collaborative researches in the community, to delineate the central community parts and main subcommunities, and to identify the main fields of interest of its members.

The rest of the paper is organized as follows. Section 2 introduces the idea of collaboration in scientific communities, presents the data used in the study and the process of establishing the co-authorship network. In Section 3, we present the results of the analysis of the co-authorship network with the emphasis on the community structure. Section 4 discusses the results, putting them in the context of related work. Finally, Section 5 draws conclusions and outlines the directions for further research.

\section{Collaboration and Scientific Community}

As mentioned in the introduction, the most important aspect of scientific community is the communication among scientists. Although there are several ways in which scientific communication can take place, presumably the most interesting pattern thereof is the collaboration among scientists. Collaboration can be described as a social process in which two or more scientists cooperate and share intellectual ideas.

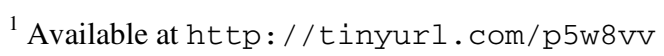


In this way, various and quite different situations can be considered to be scientific collaboration; one of them being the cooperation formalized through publishing a joint article. The cooperation of scientists in writing scientific papers, referred to as co-authorship, is in the focus of this paper and is the most frequently used for the analysis of scientific collaboration [2].

The most obvious way to represent the notion of co-authorship is the use of network, where the nodes represent individual researchers, and links among them represent co-authorship relations [2]. Once we have a network, we can apply standard social network analysis to analyze its structural properties, which will reveal important properties and structure of the corresponding scientific community. In this paper, we limit our scope of interest on one part of e-government research community that publishes papers at the International Conference on e-Government (EGOV). The analyzed data include all the papers published in all eight proceedings from the first (2002) to the last one (2009). In the continuation of this section, we introduce the data set and the corresponding co-authorship network.

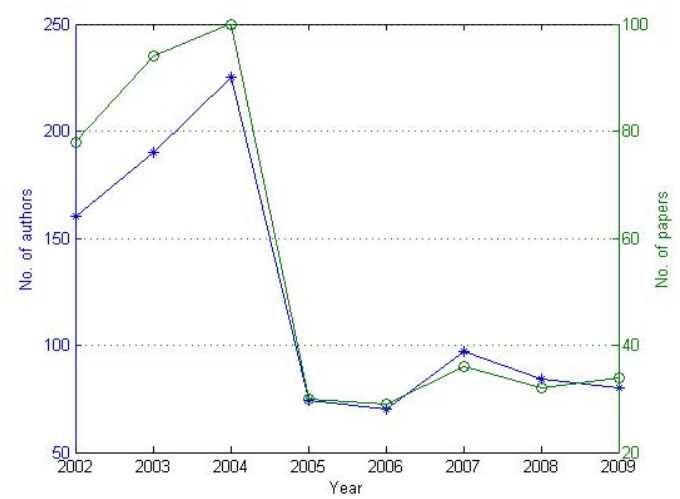

Fig. 1. Number of authors and papers published in the EGOV conference proceedings

\subsection{Data Description}

For this study, we build up on the data collected in [7], which we extended with the data about papers published in 2009. The complete data set for the period of eight years from 2002 to 2009 includes 433 papers. The total number of co-authors of these papers amount to 980 authors, out of which there are 706 different researchers.

Figure 1 summarizes the data set. It shows the significant drop of the number of published papers from about 100 in 2004 to 30 in 2005; the decrease is due to the improved review criteria and narrowed acceptance criteria. From 2005 onward, the number does not change significantly and it stays close to 30 with minor changes over the years. The change in number of authors resembles the one for papers; while in the first three years the number of authors steadily increased (from 160 to 225), fewer accepted (published) papers lead to the reduced number of authors (from 2004 to 2005 the number dropped from 224 to 74 authors). From 2005 on, it varies between slightly more than 70 to almost 100 (in 2007). 
Figure 1 shows a significant change in the data distribution from 2004 to 2005 . To avoid analysis artifacts that might be related to this abrupt change, we reduced the time-span of our analysis to the period from 2005 to 2009.

\subsection{From Data to Co-authorship Network}

The data set, described above, can be transformed to a co-authorship network following a simple procedure. For each paper in the proceedings, we collect the list of paper authors $A$. For each of them, we add a corresponding node to the network, if one has not been there yet. If the list consists of a single author we proceed with the next paper, since it does not introduces any co-authorship links in the network. Otherwise, for each pair of authors from $A$, we add an undirected link (edge) connecting the corresponding network nodes. If the link has been already present, we increase its weight by one; if the link is new, we assign weight 1 . Thus, the weight assigned to an edge connecting two nodes denotes the number of papers that the corresponding two researchers co-authored.

Following the procedure outlined above, we generated five weighted undirected co-authorship networks for each observed year of proceedings from 2005 to 2009. Using the single-year networks, we constructed five "cumulative" co-authorship networks; the first network contains data from 2005 and each successive network is built in the way that to the preceding network data from the following year is added.

Table 1. Number of nodes, edges and density of the five "cumulative" co-authorship networks

\begin{tabular}{l|rrrrr}
\hline & $\mathbf{2 0 0 5}$ & $\mathbf{2 0 0 5 - 2 0 0 6}$ & $\mathbf{2 0 0 5 - 2 0 0 7}$ & $\mathbf{2 0 0 5 - 2 0 0 8}$ & $\mathbf{2 0 0 5 - 2 0 0 9}$ \\
\hline \#nodes & 74 & 136 & 208 & 262 & 307 \\
\#edges & 91 & 170 & 295 & 395 & 467 \\
weight $=\mathbf{1}$ & 91 & 168 & 273 & 364 & 426 \\
weight $>\mathbf{1}$ & $0(0.0 \%)$ & $2(1.2 \%)$ & $22(7.5 \%)$ & $31(7.8 \%)$ & $44(9.4 \%)$ \\
Density & 0.0337 & 0.0185 & 0.0137 & 0.0116 & 0.010 \\
\hline
\end{tabular}

Table 1 presents the general properties of the five cumulative networks. The size of cumulative networks steadily increases, both in terms of the number of nodes/authors and links/co-authorships between them. The increase in size is being strictly followed by density decrease. The density of 0.01 of the final 2005-2009 network denotes that only $1 \%$ of all possible edges between nodes are present in the network. And yet, the portion of edges with weight larger than one (that is the number of research pairs that co-authored more than one article) steadily increases from initial 0 to $9.4 \%$. The increase indicates the rising collaboration interest among EGOV researchers. A reason for this trend may be due to the fact that cumulative collaboration tends to be long-term: collaborating once makes collaborating in future more likely. Note however, that individual, non-cumulative, year data (see the last column of Table 2) confirm this cumulative trend.

In the following section, we present the results of the analysis of the dynamic change of the five "cumulative" networks with the emphasis on the structure of the network for the period from 2005 to 2009. 


\section{Structure and Dynamics of the EGOV Conference Community}

Having built the cumulative co-authorship networks, we turn our attention towards analysis of the networks and results thereof. We use Pajek software tool [4] to perform the analysis of the co-authorship network. The results of the analysis are presented in three subsections. In the first one, we quantify and analyze the dynamic change of the community (network) from 2005 to 2009. Second section identifies the most active and most connected (collaborative) authors in the community. In the final, third subsection we analyze the EGOV community structure in terms of subgroups of collaborating (co-authoring) researchers, their geographical distribution, and research topics they are dealing with.

Table 2. Dynamics of the EGOV conference community from 2005 to 2009 in terms of numbers (and portions in \%) of authors (newcomers and returning) and papers (single-authored and co-authored)

\begin{tabular}{lrrrrrr}
\hline Year & \#authors & $\begin{array}{r}\text { \#new } \\
\text { authors }\end{array}$ & $\begin{array}{r}\text { \#existing } \\
\text { authors }\end{array}$ & $\begin{array}{r}\text { \#single-authrd } \\
\text { \#papers }\end{array}$ & $\begin{array}{r}\text { \#co-authored } \\
\text { papers }\end{array}$ & $\begin{array}{r}\text { papers } \\
\hline \mathbf{2 0 0 5}\end{array}$ \\
$\mathbf{2 0 0 6}$ & 74 & $74(100.0 \%)$ & 0 & 30 & $10(33.3 \%)$ & $20(66.7 \%)$ \\
$\mathbf{2 0 0 7}$ & 70 & $62(88.6 \%)$ & $8(11.4 \%)$ & 29 & $8(27.6 \%)$ & $21(72.4 \%)$ \\
$\mathbf{2 0 0 8}$ & 97 & $72(74.2 \%)$ & $25(25.8 \%)$ & 36 & $5(13.9 \%)$ & $31(86.1 \%)$ \\
$\mathbf{2 0 0 9}$ & 84 & $54(64.3 \%)$ & $30(35.7 \%)$ & 32 & $5(15.6 \%)$ & $27(84.4 \%)$ \\
\hline
\end{tabular}

\subsection{Community Dynamics}

Table 2 summarizes the results of the analysis of the EGOV community dynamics. First aspect of community dynamics is the change of the number of new researchers in the community. In the third column of Table 2, we report the number of newcomers for each year, i.e., authors that published a paper at EGOV conference for the first time in a particular year. At the beginning of the observation, in 2005, all authors are considered to be new to the community. As expected, the number of newcomers decreases through the years, from 62 newcomers in 2005 (which represents almost $20 \%$ of all the authors of the papers in the proceedings) to 45 in 2009 . The later represents only $56 \%$ of all the authors of the papers in the proceedings of the 2009 conference. The trend shows that the community reached a stage, where $44 \%$ of the authors in 2009 have already published at the same conference before, which proves the EGOV community persistence and stability.

Another aspect of community dynamics is the extent of collaboration between community members. In 2005, one third (10 out of 30 ) of the papers were written by a single author, i.e., without collaboration. This number monotonically decreased through the years, reaching the minimum of four papers $(9 \%)$ out of 34 . In other words (see the figures in the last column of Table 2) the collaboration between community members steadily increases. The increasingly complex discourse and research issues in e-government community require collaboration of larger research teams, which is reflected in the large portion (91\%) of co-authored papers. 
In sum, there is a tendency of growing collaboration among EGOV community members through the years. The tendency is accompanied by the process of community convergence and stabilization with researchers that regularly publishes at the EGOV conference.

\subsection{The Most Productive and Most Collaborative Authors}

The obvious way to identify most productive authors in the EGOV conference community is to perform frequency analysis in terms of number of papers that a community member published in the EGOV conference proceedings in the observed period. In addition, to corroborate this measure, we can also expose in how many proceedings the identified most productive authors published their papers. Table 3 presents the results of the analysis.

Table 3. The seven most productive authors in the EGOV conference community in the period between 2005 and 2009 measured in terms of number of published papers and number of conference proceedings

\begin{tabular}{lrr}
\hline Author & \#papers & \#proceedings \\
\hline Grönlund $\AA$. & 6 & 4 \\
Becker J. & 6 & 3 \\
Van Dijk J. & 5 & 5 \\
Charalabidis Y., Ferro E. & 5 & 4 \\
Andersen K.N., Niehaves B. & 5 & 3 \\
\hline
\end{tabular}

In the period 2005 to 2009 , the seven researchers, enlisted in Table 3, coauthored more than four papers in the EGOV conference proceedings. Grönlund $\AA$. and Becker J. published 6 papers, where the first author published his papers in four proceedings, and the latter published in three proceedings (in both cases, more than one paper per single-year proceedings). Each of the other five co-authored five papers. Van Dijk J. published one paper per year (five papers in five proceedings), Charalabidis Y. and Ferro E. in four proceedings, and Andersen K.N. and Niehaves B. in three.

To measure the extent to which authors collaborate with the community, we can use the co-authorship network to observe the degree of connectedness of an individual established to the other researchers in the community. To this end, social network analysis uses centrality measures for individual nodes in the network. There are three centrality measures (degree, closeness, and betweenness) which distinguish in the way the position of individual nodes within the network is referred [4]. In co-authorship network, degree centrality is equal to the number of collaborators an author has, closeness centrality indicates the accessibility (or closeness) of the observed author to the others, and betweenness centrality indicates the number of shortest paths which pass through the observed vertex of the network. Table 4 lists the most central authors of EGOV conference community wrt these three measures. 
Table 4. The most central authors in EGOV conference community in the period between 2005 and 2009 measured using degree, closeness, and betweenness centrality. Emphasized (bold) names correspond to the most central authors according to all three centrality measures, while italic names correspond to the most central authors according to two centrality measures.

\begin{tabular}{l|l|r|l|r|l|r}
\hline \multirow{2}{*}{} & \multicolumn{2}{|c|}{ Degree centrality } & \multicolumn{2}{c|}{ Closeness centrality } & \multicolumn{2}{c}{ Betweenness centrality } \\
\cline { 2 - 6 } & Author & $\%$ & Author & $\%$ & Authors & $\%$ \\
\hline 1 & Charalabidis $Y$. & 3.27 & Charalabidis $Y$. & 3.58 & Tan Y.H. & 0.11 \\
2 & Van der Geest T. & 2.94 & Van der Geest T. & 3.55 & Henriksen H.Z. & 0.10 \\
3 & Askounis D. & 2.61 & Van Dijk J. & 3.40 & Van der Geest T. & 0.10 \\
4 & Lampathaki F. & 2.61 & Arendsen R. & 3.26 & Van Dijk J. & 0.09 \\
5 & Vintar M. & 2.61 & Vintar M. & 3.07 & Vintar M. & 0.08 \\
6 & Arendsen R. & 2.29 & Askounis D. & 2.99 & Andersen K.N. & 0.07 \\
7 & Iribarren M. & 2.29 & Lampathaki F. & 2.99 & Liu J. & 0.07 \\
8 & Concha G. & 2.29 & Jansen J. & 2.90 & Arendsen R. & 0.06 \\
9 & Valdes G. & 2.29 & de Vries S. & 2.90 & Grönlund A. & 0.05 \\
10 & Solar M. & 2.29 & Gionis G. & 2.76 & Flak L.S. & 0.04 \\
11 & Van Dijk J. & 2.29 & Koussouris S. & 2.76 & Ferro E. & 0.03 \\
12 & Gionis G. & 2.29 & Tan Y.H. & 2.74 & Tarabanis K. & 0.03 \\
13 & Becker J. & 2.29 & Becker J. & 2.61 & Sein M.K. & 0.03 \\
14 & Koussouris S. & 2.29 & Iribarren M. & 2.61 & Todorovski L. & 0.02 \\
\hline
\end{tabular}

Despite the differences between the three lists, presented in Table 4, there are four authors, which occupy the central position in the community wrt all three centrality measures: van der Geest T., Vintar M., Arendsen R., and van Dijk J. The listed authors are the most central in view of their collaboration with other authors in the community, they are the most reachable (close) to other authors, and are located on the highest number of shortest paths in the network. Furthermore, van der Geest T. collaborated with 9 other authors and wrote 3 papers, Vintar M. collaborated with 8 authors in 4 different papers, Arendsen R. wrote 3 papers in collaboration with 7 other authors, and van Dijk J. wrote 5 co-authored papers in cooperation with 7 other members of EGOV conference community.

Comparison of these three lists with the list of the most productive authors from Table 3, we can see that there is a high correlation between authors' degree of collaboration with others and her/his productivity in terms of number of publications. Six out of seven authors from Table 3 (all except Niehaves B.) can be also found in at least one of the lists in Table 4, and three of them in at least two lists.

\subsection{Community Structure}

Until now, the focus of our analysis was on the properties of individual members of the observed network. In this last section, we analyze the structure of the EGOV community network as a whole. To this end, we apply methods for identifying components and cores in the network that help us identify clusters of highly interconnected (collaborative) subgroups of the EGOV conference community.

The idea of finding components in co-authorship network arises from the presumption that the actors in a network compose a sub-group (component) in which every member of the component can be reached from all other members of the same component. Considering the definition, in co-authorship network components should 
reveal such groups of authors which collaborate frequently and, presumably, share common research topic(s). On the other hand, the search for cores implies even more restricted conditions under which actors represent the core sub-group of the community, as each of its members is linked with all the other members of the same core sub-group. In this sense, one core-group actually represents a set of paper(s) in which all core members collaborated [5].

Table 5. The analysis of the nine components of the EGOV co-authorship network. Each component corresponds to a research group that co-authored at least three joint papers.

\begin{tabular}{|c|c|c|c|c|}
\hline \multicolumn{2}{|c|}{$\begin{array}{l}\text { \# authors/ } \\
\text { papers }\end{array}$} & \multirow{2}{*}{$\frac{\text { Representative }}{\text { Van Dijk J. }}$} & \multirow{2}{*}{$\begin{array}{l}\text { Geographical } \\
\text { distribution } \\
\text { Netherlands }\end{array}$} & \multirow{2}{*}{$\begin{array}{l}\text { Thematic topics } \\
\text { citizen-centric e-services; user } \\
\text { profiling; delivery channels; e-services } \\
\text { adoption and usage }\end{array}$} \\
\hline 16 & 9 & & & \\
\hline 15 & $\overline{9}$ & $\begin{array}{l}\text { Andersen K.N. } \\
\text { Tan Y.H. }\end{array}$ & $\begin{array}{l}\text { Denmark, } \\
\text { Norway, } \\
\text { Netherlands }\end{array}$ & $\begin{array}{l}\text { project evaluation; benefits of IT usage; } \\
\text { e-customs; super-national e-services }\end{array}$ \\
\hline 12 & 7 & Vintar M. & $\begin{array}{l}\text { Slovenia, } \\
\text { Greece }\end{array}$ & $\begin{array}{l}\text { indicators of e-government } \\
\text { development; e-services adoption and } \\
\text { usage; life events and integration of e- } \\
\text { services }\end{array}$ \\
\hline 10 & $\overline{7}$ & Grönlund $\AA$. & $\begin{array}{l}\text { Sweden, } \\
\text { Norway }\end{array}$ & e-government research analysis; misc \\
\hline 9 & 7 & Becker J. & Germany & misc \\
\hline 8 & $\overline{6}$ & Ferro E. & Italy, USA & digital divide and IT literacy \\
\hline 11 & 5 & Charalabidis Y. & Greece & $\begin{array}{l}\text { interoperability; meta-data and } \\
\text { (semantic) annotation of e-services }\end{array}$ \\
\hline 5 & 4 & Mentzas G. & Greece & evaluating quality of e-services \\
\hline 7 & 3 & $\begin{array}{l}\text { Corradini F. } \\
\text { Sabucedo L.A. }\end{array}$ & Italy, Spain & $\begin{array}{l}\text { semantic-driven integration of e- } \\
\text { services }\end{array}$ \\
\hline
\end{tabular}

In our co-authorship network, we can identify 82 components. Out of these, 13 components contain only one author; the 13 components correspond to isolated authors that never co-authored a paper with others. On the other side of the spectrum is the largest component consisting of 16 authors. Note however, that here we can perceive the impact of papers with significantly higher number of authors compared to other papers: namely, in such a situation a single paper co-authored by many authors would induce a (non-)representative component. To overcome this problem, we augment each component with the list corresponding papers and consider only those components that are induced by at least three joint publications. In addition, we also filter out all the components with less than five researchers.

Table 5 presents the results of the component analysis by enlisting all nine components that satisfy the three-joint-papers criterion explained above. Each community subgroup is described with the leading researcher that is a co-author of majority of the papers in the component, the geographical distribution of authors' affiliations, and the list of thematic topics of the papers in the component. 
The results show that most (all but the representatives of the last two components) of the components representatives were already identified as most productive or most central ones. Furthermore, most of the identified sub-groups have narrow geographical distribution: seven out of eight components are entirely from Europe, five are very even tighter, including single region or country, or, in some cases, a single institution. International or trans-Atlantic collaboration is relatively rare. Finally, there is a great variety of thematic topics addressed by the researchers in different groups. Note also that the identified sub-communities are orthogonal in the topics they deal with; each of them develops its own (relatively narrow) expertise area that is different from others. In very rare cases, the identified group covers a wide range of thematic topics marked as miscellaneous in Table 5.

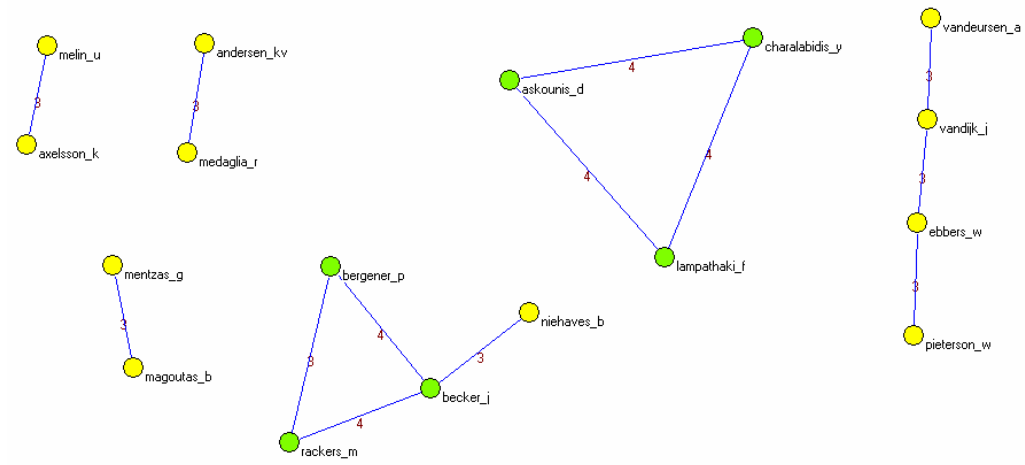

Fig. 2. Six cores of the EGOV co-authorship network

In the continuation of the analysis, we applied the same criterion as above, that is, we took into account only those collaborations that resulted in at least three joint publications. Figure 2 depicts the six cores of the EGOV co-authorship network that follow the three-joint-papers criterion. The identified cores confirm the findings of the component analysis: each of the five cores corresponds to one of the identified components from Table 5. The sixth core (Melin U. and Axelsson K.) identifies a Sweden sub-community of two co-authors of three papers, two of them being citizen participation and involvement in e-government projects. Another fact can be derived from the result depicted in Figure 2: only these 17 authors (out of 307; 5\%) have been involved in more than two joint collaboration ventures. This is another piece of evidence that the long-term collaboration within the EGOV conference community is relatively rare.

In sum, the structural analysis of the EGOV community shows that a number of sub-communities has emerged, each of them dealing with topics that are orthogonal to the thematic topics of the other. Virtually all the sub-communities have a representative researcher that is ranked among top active or collaborative community members. Most of the sub-communities have narrow geographical distribution including a single region, country or even institution. The community members are mostly affiliated at institutions in European countries, and large-scale international collaborations are very rare. 


\section{Discussion}

As we emphasized in the introduction, there are number of studies that analyze the state-of-the-art and the dynamics of the development of the e-government research (EGR) field $[1,8,6,7,9,10,14,17]$.

Our study differs from them in several ways. First, it relies on a standard scientometric method widely used to analyze other research fields. Only the study [7] applies the same analysis methodology to the task of analyzing citation networks; in this paper we deal with analysis of co-authorship networks and the EGOV conference scientific community. Note however that other authors have been using the same methodology in various e-government studies, see e.g., analysis of partnership networks for implementing an e-government project [4], implementing local e-government policy [12], or coordination of soft-target organizations [16]. All these studies combine social network analysis with other qualitative and quantitative methods, such as interviews and surveys. In our study, we do not need these, since we collect the empirical data systematically from all the papers published in the proceedings of the EGOV conference in the last five years. The systematic data collection is the second distinguishing property of our study from the others. We focus the analysis on systematic data from a single publication venue instead of using a sample of data about articles and papers from various publication venues. Although this decision makes the definition and the scope of the scientific community clear (as opposed to the vague definition from [14]), it can also be regarded as a main limitation of our study, which we further discuss in the final section.

The present paper offers the findings of co-authorship network analysis which is latterly used as a proxy for the study of collaboration [2]. We should note however, that there exist limitations related to the study of scientific collaboration through publications and to the bibliometric studies in general. On one hand, we should consider the practice of making colleagues or superiors "honorary co-authors" for purely social reasons. On the other hand, scientific collaboration does not necessarily lead to co-authored papers.

Finally, the comparison of our results with the results of our citation analysis [7] provides several insights. First, when comparing the list of authors mention there, we find out that only two most cited authors (i.e., Grönlund $\AA$. and Van Dijk J.) are confirmed to be members of the EGOV community as defined in this paper. Thus, most influential authors and literature come from outside EGOV community. This comes by no surprise, if we consider another result reported in [7], that only $8.5 \%$ of citations in the EGOV conference papers refer to other EGOV conference papers. Second, note that the thematic topics identified in this paper match the most influential (most cited) topics identified in [7], i.e., EGR analysis, seamless citizencentric integration of e-services, interoperability, influence of information technology (IT) on organizational change, digital divide and IT literacy, user profiling and personalization of e-services. 


\section{Conclusion}

Scientific community is a conglomerate of scientists who spread and diffuse their knowledge mainly through the publication of their theories and studies. In the core of every analysis of such communities is the study of communication patterns among scientists on the basis of citation, co-citation, or co-authorship network analysis. In the present paper, we focused on the analysis of EGOV conference community as one relatively small albeit representative part of the whole egovernment research (EGR) community. We applied social network analysis to study the co-authorship network and linked the findings with the results of citation network analysis performed on the same data. This enabled us to improve the map of e-government research field with identification of the community most active and central authors, community structure and its core sub-groups. Beside with researchers' membership these sub-communities are characterized with the geographical distribution of the core authors and thematic topics prevailing in the EGR community.

However, we cannot disregard the fact that the data used to perform the analysis is far from being exhaustive. The first obvious limitation is its focus on a single publication venue that is the proceedings of the EGOV conference. Although the use of data on a single publication venue in this paper is well considered since the aim of the present paper was to complete the map of EGR, we are aware of the need for the extension of the data set with data on papers from other e-government conferences (such as the annual conference the Digital Government Society of North America) and journals (e.g., Government Information Quality and Information Polity).

In the present paper we focused on the co-authorship networks, where nodes represent authors and edges represent joint articles published in the EGOV conference proceedings. Usage of social network analysis methods, however, allows for establishment and analysis of other types of networks as well, on which we will focus in our future work. One possibility is the analysis of the citation networks considering publication venues where the referenced papers come from. Such analysis would reveal the most influential "neighboring" scientific fields and publication venues with highest impact on the development and shape of the EGR field. Furthermore, revising the definition of nodes and the meaning of relations among them opens even more analysis opportunities where other networks (e.g., networks where nodes correspond to publication units or where relations correspond to co-citations) can reveal some other structural patterns and characteristics of the observed EGR field. Note however, that it will be quite a challenge to uniquely identify publication units or co-citations in the rather "noisy" data set of references. Ultimately, there is a challengeable issue of integrating the results of analyzing different networks of relations among scientists and/or publication venues into a unified map of the EGR field.

Acknowledgments. This material is based upon work supported by the Slovenian Research Agency through the funds for training and financing young researchers. Thanks to the four anonymous reviewers for their valuable comments on an earlier version of the manuscript. 


\section{References}

1. Andersen, K.V., Henriksen, H.Z.: The First Leg of E-Government Research: Domains and Applications Areas 1998-2003. International Journal of Electronic Government Research 1(4), 26-44 (2005)

2. Bordons, M., Gómez, I.: Collaboration Networks in Science. In: Cronin, B., Atkins, H.B. (eds.) The web of knowledge: a festschrift in honor of Eugene Garfield. ASIS Monograph Series. Information Today, Medford (2001)

3. Claussen, H., Wormell, I.: A bibliometric analysis of IOLIM conferences 1977-1999. Journal of Informaztion Science 27(3), 157-169 (2001)

4. Cotterill, S., King, S.: Public Sector Partnerships to Deliver Local E-Government: A Social Network Study. In: Wimmer, M.A., Scholl, H.J., Grönlund, A. (eds.) EGOV 2007. LNCS, vol. 4656, pp. 240-251. Springer, Heidelberg (2007)

5. de Nooy, W., Mrvar, A., Batagelj, V.: Exploratory social network analysis with Pajek. Cambridge University Press, New York (2005)

6. Dwivedi, Y.K.: An analysis of e-Government research published in Transforming Government: People, Process and Policy (TGPPP). Transforming Government: People, Process and Policy 1(3), 7-15 (2009)

7. Erman, N., Todorovski, L.: Mapping the E-Government Research with Social Network Analysis. In: Wimmer, M.A., Scholl, H.J., Janssen, M., Traunmüller, R. (eds.) EGOV 2009. LNCS, vol. 5693, pp. 13-25. Springer, Heidelberg (2009)

8. Grönlund, Å., Andersson, A.: e-Gov Research Quality improvements Since 2003: More Rigor, but Research (Perhaps) Redefined. In: Wimmer, M.A., Scholl, H.J., Grönlund, Å., Andersen, K.V. (eds.) EGOV 2006. LNCS, vol. 4084, pp. 1-12. Springer, Heidelberg (2006)

9. Grönlund, A.: State of the Art in eGov Research - A Survey. In: Traunmüller, R. (ed.) EGOV 2004. LNCS, vol. 3183, pp. 178-185. Springer, Heidelberg (2004)

10. Heeks, R., Bailur, S.: Analyzing e-government research: Perspectives, philosophies, theories, methods, and practice. Government Information Quarterly 24, 243-265 (2007)

11. Mali, F.: Znanost kot sistemski del družbe (Science as a systematic part of society). Faculty of social sciences, Ljubljana (1994)

12. Medaglia, R.: Local Networking for e-Services: A UK Case Study. In: Wimmer, M.A., Scholl, H.J., Grönlund, Å., Andersen, K.V. (eds.) EGOV 2006. LNCS, vol. 4084, pp. 256-268. Springer, Heidelberg (2006)

13. Otte, E., Rousseau, R.: Social network analysis: a powerful strategy, also for the information sciences. Journal of Information Science 28(6), 441-453 (2002)

14. Scholl, H.J.: Profiling the EG Research Community and Its Core. In: Wimmer, M.A., Scholl, H.J., Janssen, M., Traunmüller, R. (eds.) EGOV 2009. LNCS, vol. 5693, pp. 1-12. Springer, Heidelberg (2009)

15. Scientometrics and bibliometrics,

http://www.sciencemetrix.com/eng/ methods-_scientometrics_t.htm (accessed, February 2009)

16. Uddin, M.S., Hossain, L.: Towards coordination preparedness of soft-target organisation. In: Wimmer, M.A., Scholl, H.J., Janssen, M., Traunmüller, R. (eds.) EGOV 2009. LNCS, vol. 5693, pp. 54-64. Springer, Heidelberg (2009)

17. Yildiz, M.: E-government research: Reviewing the literature, limitations, and ways forward. Government Information Quarterly 24, 646-665 (2007) 